\title{
Étude expérimentale sur la reproduction chez le cobaye (Cavia porcellus) élevé en zone périphérique de Kinshasa, R D Congo
}

\author{
J M Umba, JC K Kashala*, F AB Khand'Mate*, R L N Ipungu*, A et D P Khasa** \\ Centre de promotion et de diffusion de mini-élevages, Institut Supérieur Agro-Vétérinaire (ISAV) de Kimwenza \\ *Faculté de Médecine Vétérinaire, Université de Lubumbashi, B.P. 1825 Lubumbashi, République Démocratique du \\ Congo \\ ${ }^{* *}$ Centre d'étude de la forêt, Faculté de Foresterie et de Géomatique, Université de Laval, Québec, Canada \\ joachimumba@yahoo.fr et jckkashala@homail.fr
}

Original submitted in on $2^{\text {nd }}$ August 2017. Published online at www.m.elewa.org on $30^{\text {th }}$ September 2017 https://dx.doi.org/10.4314/iab.v117i1.9

\section{RÉSUMÉ}

Objectif : Une étude expérimentale sur les paramètres de la reproduction chez le cobaye (Cavia porcellus) élevé en zone périphérique de Kinshasa a été réalisée. Son objectif est d'apprendre aux éleveurs des cobayes une gestion rationnelle de la reproduction par un choix judicieux des femelles actives à la saillie, de poids et âge requis et leur accouplement aux mâles performants (locaux ou exotiques) afin d'assurer une meilleure productivité

Méthodologie et résultats: L'étude a porté sur 216 géniteurs dont 36 mâles et 180 femelles venant respectivement de la République Démocratique (Bukavu et Kinshasa) et de la Belgique, qui ont donné 1002 cobayaux après divers croisements. Le poids vif moyen à l'âge zéro pour les deux sexes est de 70,49 \pm $20,52 \mathrm{~g}$; il est sous l'influence du poids de leur mère et varie selon l'importance de la nichée. Le poids vif moyen au sevrage à 3 semaines est de 127,45 $\pm 49,48 \mathrm{~g}$; il a une nette répercussion sur la croissance des animaux. Les moyennes de taille de nichée varient entre 1,73 et 3,10, suivant l'origine des parents. L'intervalle entre mises bas varie entre $89,3 \pm 4,3$ jours et $90,2 \pm 5,5$ jours suivant l'origine des parents ; la différence n'est pas significative. L'âge moyen à la 1ère saillie varie de $154,7 \pm 30,3$ jours à $228,5 \pm 28,2$ jours ; il y a des fortes variations suivant l'origine des parents.

Conclusion et application des résultats : L'éleveur des cobayes doit se choisir les femelles d'âge et poids requis, aptes à la saillie, afin d'assurer une reproduction régulière et rationnelle ; mais il doit aussi se choisir des femelles en bonne santé afin de les accoupler aux mâles performants locaux ou exotiques pour améliorer leur productivité.

Mots clés : cobaye, paramètres de reproduction, Kinshasa, R D Congo 


\section{Experimental study on breeding guinea pigs (Cavia porcellus) raised in peripheral area of Kinshasa,} Congo R D

Objective: An experimental study on reproductive parameters in guinea pigs (Cavia porcellus) raised in the peripheral area of Kinshasa was carried out. Its objective is to teach ruminant management of rational reproduction by a judicious selection of the active females at the breeding, of the required weight and age and their coupling to the performing males (local or exotic) in order to ensure a better productivity.

Methodology and results: The study included 216 sires including 36 males and 180 females from the Democratic Republic of Congo (respectively Bukavu and Kinshasa) and Belgium, who gave young guinea pigs 1002 after various crosses. The average live weight at birth for both sexes was $70.49 \pm 20.52 \mathrm{~g}$; it is influenced by the weight of their mother and varies depending on the size of the litter. The average live weight at weaning at three weeks is $127.45 \pm 49.48 \mathrm{~g}$; has a distinct effect on the growth of animals. The average size of the litter varies between 1.73 and 3.10 , depending on the origin of the parents. The interval between calving ranges from $89.3 \pm 4.3$ days and $90.2 \pm 5.5$ days following the origin of the parents ; the difference is not significant. The average age at the first calving ranges from $154.7 \pm 30.3$ days to $228.5 \pm 28.2$ days ; there are strong variations according to the origin of the parents.

Conclusion and application of results: The breeder of the guinea-pigs must select the females of suitable age and weight, suitable for breeding, in order to ensure a regular and rational breeding; but must also choose healthy females in order to mate them to local or exotic performing males to improve their productivity.

Keywords : guinea pig, reproductive parameters, Kinshasa, Congo R D

\section{INTRODUCTION}

En République Démocratique du Congo, le problème de sécurité alimentaire se pose avec acuité eu égard à l'inadéquation entre le disponible alimentaire et une population de plus en plus galopante. Une des raisons de cette insuffisance alimentaire est étroitement liée aux systèmes d'exploitation agricoles au sens large. Dans le domaine de l'élevage, plus spécialement en caviaculture, la faible productivité des animaux est en grande partie liée au mode d'élevage qui est du type familial et qui se pratique traditionnellement à l'intérieur des habitations. On y observe l'inexistence de gestion de la reproduction. La saillie qui se fait naturellement, ne tient pas compte de certains facteurs qui peuvent non seulement se traduire par des dégénérescences entraînant des mortinatalités et des dystocies, mais également compromettre la vie sexuelle des femelles. En effet, la reproduction normale et régulière représente la base essentielle de la rentabilité de l'élevage. II y a lieu économiquement de rechercher l'efficacité de chaque accouplement. Un nombre non négligeable de femelles est considéré comme subfertiles voire même stériles et éventuellement réformées alors que cet état est uniquement la résultante de la part de l'éleveur d'une méconnaissance des conditions optimales d'exploitation. La connaissance des facteurs interférant sur la reproduction est donc essentielle pour veiller à maintenir un taux de fertilité optimum (Dérivaux et Ectors 1986). II apparaît que les individus ne sont aptes à se reproduire qu'à partir $\mathrm{du}$ moment où les organes génitaux sont suffisamment développés et physiologiquement aptes à fonctionner ; ces conditions sont fonction de l'âge et surtout du degré de développement des individus (Dérivaux et Ectors 1986). Pour toutes ces raisons, il nous est paru opportun, compte tenu du système de la caviaculture où les animaux de tous âges sont mélangés, de voir quel pourrait être l'impact de l'âge et du poids des femelles à la saillie sur leurs performances de reproduction, lorsque les conditions d'alimentation sont bonnes. Dans les conditions physiologiques, plusieurs facteurs peuvent avoir une influence sur les performances de reproduction de la femelle. Parmi ces facteurs, on distingue l'âge, les facteurs génétiques, l'environnement et l'alimentation. Notre étude se focalise sur les aspects de la reproduction en exploitant :

L'amélioration de la productivité des animaux par une gestion rationnelle de la reproduction ; c'est-àdire : 
Identifier les sujets les plus aptes à la reproduction ; Identifier les provenances des cobayes qui produisent plus de chair ;

Déterminer l'âge et le poids auxquels ces sujets sont le plus actifs à la saillie ;

Utiliser les sujets sélectionnés dans l'amélioration de la productivité de l'élevage de cobayes. En Amérique du Sud, il existe des élevages de cobaye basés sur les principes modernes de production animale: pesées régulières, contrôle de reproduction et du sevrage, suivi de la croissance et de

\section{MATÉRIEL ET MÉTHODES}

Milieu : Les expériences se sont déroulées au Centre de promotion et de diffusion des Mini-élevages de l'Institut Supérieur Agro-vétérinaire (ISAV) Kimwenza dans la commune de Mont-Ngafula, ville province de Kinshasa. La température et l'humidité relative durant les expériences ont varié respectivement de 20 à $30^{\circ} \mathrm{C}$ et de 80 et $85 \%$. La pluviométrie moyenne annuelle est de $1100 \mathrm{~mm}$. Les températures et les précipitations moyennes des vingt dernières années de Kinshasa sont représentées dans la courbe ombrothermique ci-dessous. Le climat est de type tropical $\mathbf{A w}_{4}$, selon Köppen, (1936). l'engraissement, sélection appropriée des sujets d'élevage. La connaissance de la caviaculture n'est pas une activité récente en R.D.C. Le cobaye est prolifique et a une grande vitesse de croissance. Cependant, en Afrique, cet élevage est pratiqué de façon traditionnelle, à l'intérieur des cuisines. Suite à la dégénérescence des races locales, notre travail se veut une contribution à la sélection et à l'amélioration génétique des cobayes pour augmenter la productivité des élevages caviacoles locaux.

Les cobayes sont adaptés à ce climat pour leur physiorégulation et l'intervalle de variations physiologiques est de $37,8^{\circ} \mathrm{C}$ et $39,5^{\circ} \mathrm{C}$. Le sol est sablonneux, avec une végétation des graminées (Hyparrhenia sp., Trypsacum laxum, Panicum maximum) et des légumineuses (Pueraria javanicum, Desmodium intortum). A Kinshasa, il existe une longue saison des pluies de 8 mois qui va d'octobre à mai, entrecoupée d'une courte petite saison sèche entre janvier et février, et une saison sèche qui s'étale de juin à septembre.

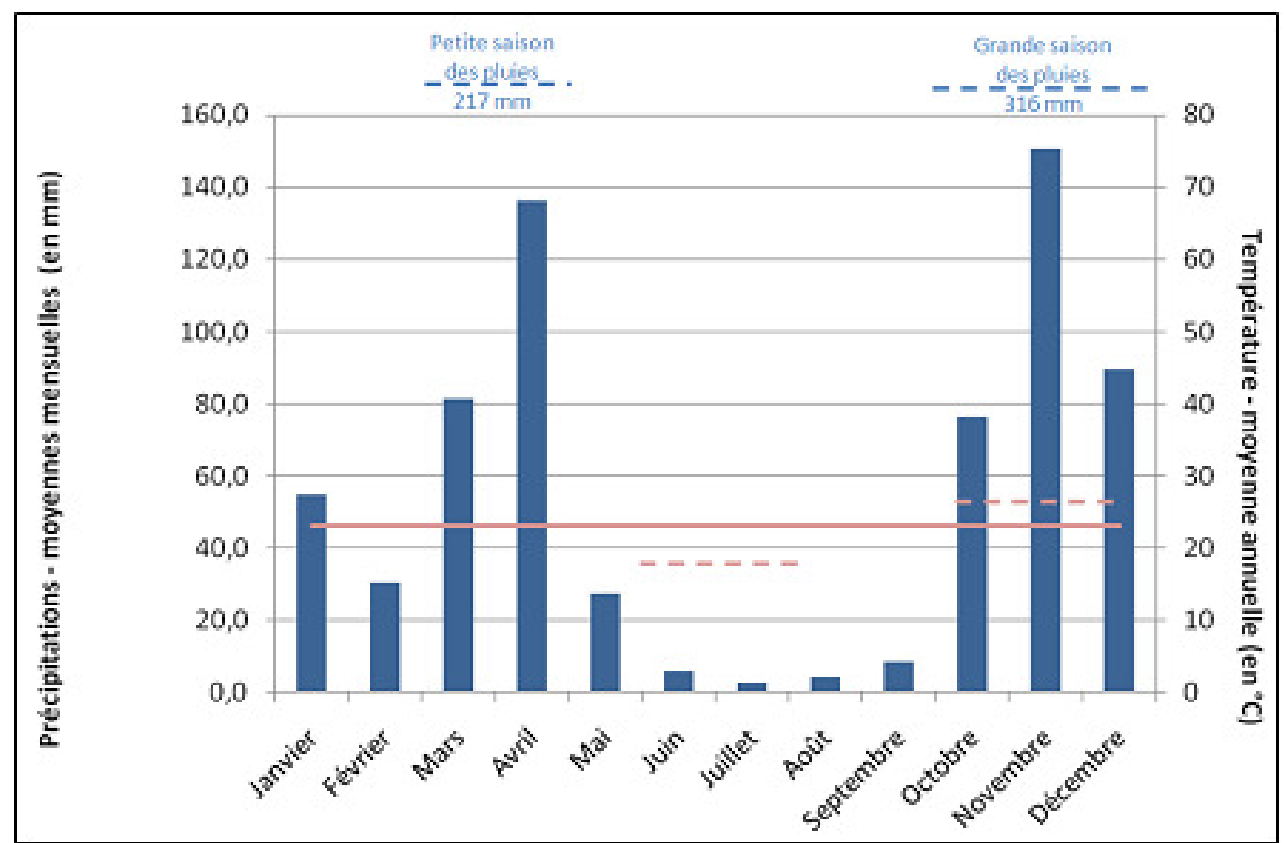

Figure 1 : Précipitations moyennes mensuelles (Service météo Binza Ozone, 2011) 


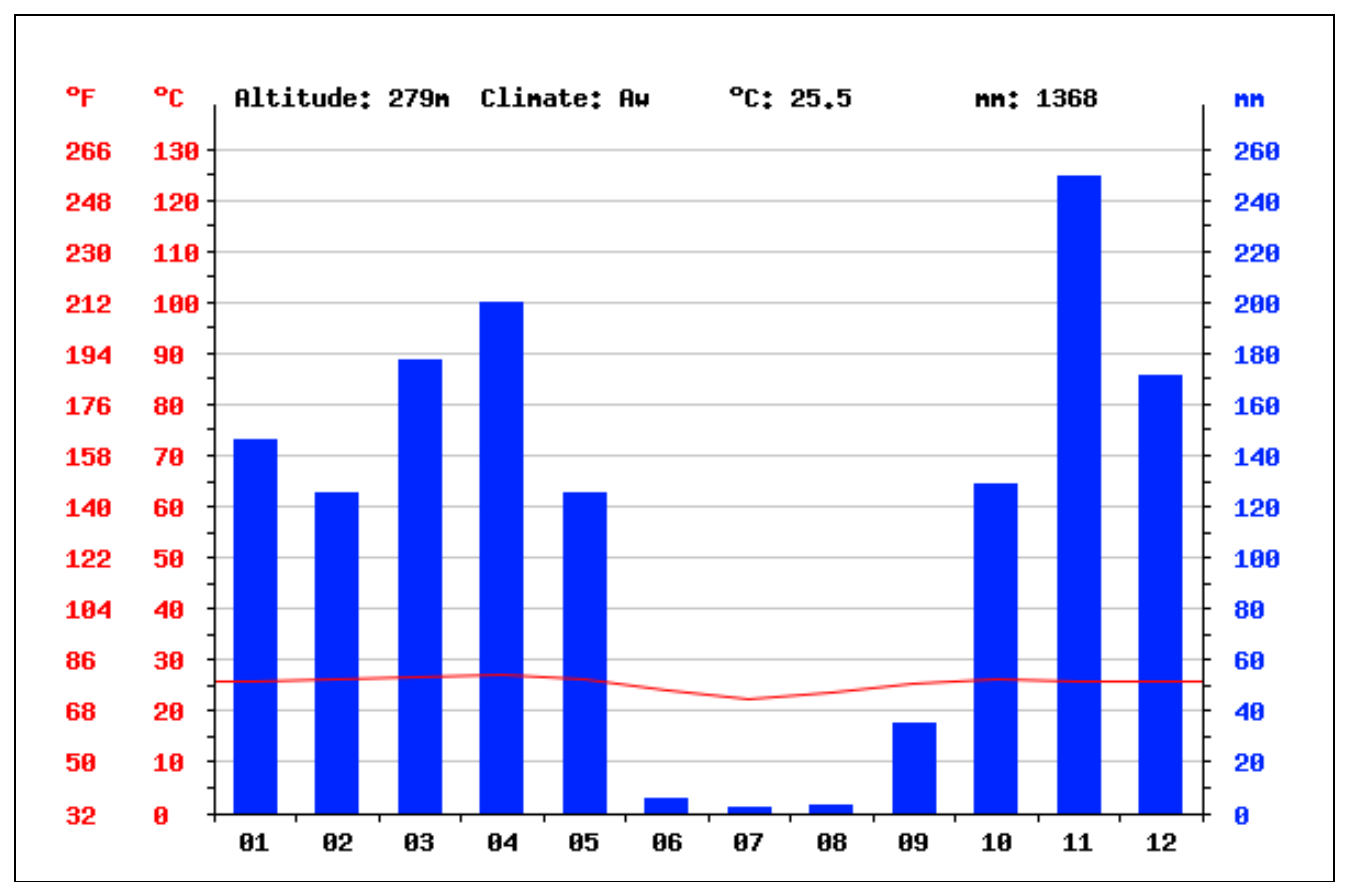

Source : www.google.cd

Figure 2: Températures moyennes mensuelles (Service météo Binza Ozone, 2011)

Kimwenza est située à la périphérie sud-ouest de l'agglomération de Kinshasa et s'étend sur les versants de part et d'autre de la Lukaya à $8 \mathrm{~km}$ de l'Université de Kinshasa. Kimwenza est administrativement un quartier du Mont-Ngafula, une des Communes de la ville de Kinshasa.

Matériel animal : 216 géniteurs dont 36 mâles et 180 femelles ayant donné naissance après accouplements à 1002 cobayaux ont servi comme matériel d'expérimentation.

Méthodes : Les 36 mâles et 180 femelles ont été croisés à raison de 5 femelles par mâle. Trois générations d'accouplement ont été effectuées tenant compte de 68 jours de gestation et deux semaines de lactation. Le dispositif expérimental utilisé était donc le modèle

\section{RÉSULTATS}

Les performances de reproduction ont porté sur l'âge à la première mise bas, l'intervalle entre les mises bas, la aléatoire hiérarchisé ; 1002 cobayaux ont été obtenus de ces accouplements. A chaque mise-bas, les caractères zootechniques prélevés, qui ont servi de variables dépendantes pour l'analyse des données, sont les suivants :

- L L'âge à la première mise bas ;

- L'intervalle entre les mises bas ;

- La taille de nichées à la naissance par génération ;

- Le poids à la naissance ;

- Le poids au sevrage.

Les analyses statistiques ont été réalisées au moyen du logiciel Excel pour les moyennes et les écart-types des données.

taille de nichées à la naissance par génération, le poids à la naissance et le poids au sevrage.

\section{Croisement ${ }^{\top}$ Bukavu $\mathrm{X} \odot$ Bukavu}

Tableau 1 : Données moyennes de reproduction des cobayes issus des croisements Bukavu X Bukavu

\begin{tabular}{|c|c|c|c|c|c|c|c|}
\hline \multirow{3}{*}{$\mathrm{n}=\mathbf{2 0}$} & \multicolumn{2}{|c|}{ Taille de nichée } & \multicolumn{2}{|c|}{ Poids à la naissance } & \multicolumn{2}{|c|}{ Poids au sevrage } & \multirow{2}{*}{$\begin{array}{c}\text { Intervalle } \\
\text { mise bas } F_{1-} \\
F_{2}\end{array}$} \\
\hline & $F_{1}$ & $F_{2}$ & $F_{1}$ & $F_{2}$ & $F_{1}$ & $F_{2}$ & \\
\hline & $2,6 \pm 0,8$ & $2,95 \pm 1,1$ & $\begin{array}{c}83,1 \pm \\
17,9\end{array}$ & $80,91 \pm 17,9$ & $\begin{array}{r}145,0 \\
\pm 40,4\end{array}$ & $137,80 \pm 46,3$ & $90,20 \pm 5,5$ \\
\hline
\end{tabular}


La taille de nichée pour le croisement ${ }^{\top}$ Bukavu $\mathbf{x}$ Q Bukavu en $F_{1}$ le plus élevée est 4 cobayaux et la plus faible est 1 , tout comme pour le $F_{2}$. Le poids à la naissance le plus élevé à la naissance en $F_{1}$ est de $125 \mathrm{~g}$ et en $F_{2}$ est de $110 \mathrm{~g}$; au sevrage il est de $260,3 \mathrm{~g}$ en $F_{1}$ et en $F_{2}$ il est de $270 \mathrm{~g}$. L'intervalle des mises bas est de $90,20 \pm 5,5$ jours.

Croisement $\stackrel{\curvearrowright}{ }$ Belgique $\mathbf{X}$ Kinshasa

Tableau 2. Données moyennes de reproduction des cobayes des croisements Belgique $X$ Kinshasa

\begin{tabular}{|c|c|c|c|c|c|c|c|}
\hline \multirow{3}{*}{$\mathrm{n}=\mathbf{2 0}$} & \multicolumn{2}{|c|}{ Taille nichée } & \multicolumn{2}{|c|}{ Poids à la naissance } & \multicolumn{2}{|c|}{ Poids au sevrage } & \multirow{2}{*}{$\begin{array}{c}\text { Intervalle mise } \\
\text { bas F1-F2 }\end{array}$} \\
\hline & F1 & F2 & F1 & F2 & F1 & F2 & \\
\hline & $2,8 \pm 0,6$ & $2,8 \pm 0,7$ & $\begin{array}{r}79,7 \pm \\
14,7\end{array}$ & $72,4 \pm 15,0$ & $\begin{array}{r}155,6 \\
\pm 39,2\end{array}$ & $\begin{array}{r}123,9 \pm 3 \\
6,5\end{array}$ & $89,3 \pm 4,3$ \\
\hline
\end{tabular}

La taille de nichée pour le croisement 3 Belgique $\mathbf{x}$ Kinshasa en $\mathrm{F}_{1}$ le plus élevée est 4 cobayaux et la plus faible est 1 , tout comme pour le $F_{2}$. Le poids à la naissance le plus élevé à la naissance en $F_{1}$ est de 123,1 $g$ et en $F_{2}$ est de $125,2 \mathrm{~g}$ au sevrage il est de $203 \mathrm{~g}$ en $\mathrm{F}_{1}$ et $F_{2}$ il est de $270 \mathrm{~g}$. L'intervalle des mises bas est de $89,3 \pm 4,3$ jours.

Tableau 3 : Âge moyen à la 1ère mise bas (en jours)

\begin{tabular}{c|c}
\hline${ }^{\wedge}$ Belgique $\mathbf{X} \odot$ Kinshasa & \multicolumn{1}{|}{ Bukavu $\mathbf{X} \odot$ Bukavu } \\
\hline $\mathbf{2 2 0 , 4} \pm \mathbf{2 7 , 5}$ & $228,5 \pm 28,2$ \\
\hline
\end{tabular}

Au regard de ce tableau, nous constatons que l'âge moyen à la 1ère mise base de Bukavu X Bukavu est plus élevé que celui de Belgique X Kinshasa.

Tableau 4 : Âge moyen de la 1ère saillie (en jours)

\begin{tabular}{c|c}
\hline Belgique $\mathbf{X} \odot$ Kinshasa & \multicolumn{1}{|}{ Bukavu $\mathbf{X} \odot$ Bukavu } \\
\hline $\mathbf{1 5 4 , 7 \pm 3 0 , 3}$ & $169,25 \pm 22,8$ \\
\hline
\end{tabular}

L'âge moyen de la 1ère saille de Bukavu X Bukavu est plus élevée que celui de Belgique $X$ Kinshasa. La

\section{DISCUSSION}

Évolution du poids vif de la naissance au sevrage : Le poids vif moyen à l'âge zéro pour les deux sexes est de $70,49 \pm 20,52 \mathrm{~g}$. Le poids à la naissance des petits est sous l'influence du poids de leur mère. Selon l'ordre et l'importance de la nichée, le poids peut varier à la naissance de 80 à $100 \mathrm{~g}$ dans les conditions expérimentales et $150 \mathrm{~g}$ en élevage rationnel (Hardouin et Thys 1997). Les moyennes renseignées par Fosto et al (1995) sont de 85,2 $\pm 15,5 \mathrm{~g}$. Si nos résultats sont inférieurs à ceux préconisés par Hardouin et Thys (1997), cependant ils avoisinent ceux de Fosto et al (1995) au Cameroun. Ces fortes variations observées sont probablement dues aux modes d'élevage et aux conditions de notre élevage qui est encore de type traditionnel. L'alimentation non équilibrée peut constituer un autre facteur pour ces faibles poids. Le poids vif différence entre l'âge de la 1ère saille et l'âge de la 1ère mise bas entre les deux croisements n'est pas significatif.

moyen au sevrage à 3 semaines est de 127,45 \pm 49,48 $\mathrm{g}$; on observe une forte variation de ce poids due probablement à une intense croissance et à la compétitivité lors de la prise de lait par les petits pour les femelles dont l'effectif de la nichée est importante. L'âge au sevrage a une nette répercussion sur la croissance des animaux. Fosto et al (1995) donnent des valeurs de l'ordre de $161,1 \mathrm{~g}$ pour le poids au sevrage à 3 semaines au Cameroun ; ces données avoisinent les nôtres. Salsac (2004) estime qu'il faut sevrer si possible à une semaine, plutôt qu'à deux ou à trois semaines car la courbe de lactation de la mère chute après 5 jours ; on obtient de bons gains de poids avec des concentrés. Le poids est un paramètre plus significatif que l'âge pour choisir les reproducteurs ; on attendra que la femelle ait atteint un poids de $500 \mathrm{~g}$ vers 3 mois pour le premier 
accouplement. L'alimentation et la génétique jouent un rôle très important sur la productivité du cobaye (Chauca 1999 ; Bauer et al 2009). Selon Bauer et al (2009), la croissance est sensible à la qualité du régime pré et postnatal maternel.

Taille de la nichée : Les moyennes de nichée d'origine de la femelle oscillent pour tous les cas étudiés entre 1,73 et 3,10. L'effectif le plus faible a été enregistré avec la femelle de Mbanza-Ngungu $(1,73 \pm 0,45)$ et le plus élevé avec celle de la Belgique $(3,10 \pm 0,66)$. Toutefois, il y a lieu de signaler l'effectif réduit des mâles et femelles de Kisantu et de Mbanza-Ngungu. En considérant les origines des parents mâle et femelle, les valeurs en taille de nichée des parents en provenance de Belgique et de Bukavu sont excellentes, ils donnent le nombre de petits le plus élevé soit respectivement 3,10 et 2,81. Les femelles et mâles en provenance de Kinshasa et de Mbanza-Ngungu donnent des nichées dont les nombres moyens de petits sont respectivement de 2,34 pour les premiers et 2,17 pour les seconds. Les données de la littérature renseignent des tailles de nichée allant de 1 à 6 petits, pour une moyenne variant entre 2,8 et 3,4 , cela malgré l'existence de 2 tétines seulement; il y a moins de jeunes lors de deux premières gestations (Cicogna 2000; Hardouin 2000; Havrez 2002; Salsac, 2004). Nous avons obtenu des tailles moyennes de nichées importantes (2,8 à 3,1 ) surtout pour les parents en provenance de la Belgique. La taille de la nichée peut influencer le poids à la naissance, comme signalé ci-haut (Hardouin et Thys 1997). Fosto et al (1995) donnent des tailles de nichées

\section{CONCLUSION}

La reproduction est un élément clé de l'élevage qu'il est nécessaire de maîtriser au mieux afin d'assurer une bonne productivité. L'éleveur des cobayes doit se choisir les femelles d'âge et poids requis, aptes à la saillie, afin d'assurer une reproduction régulière et rationnelle ; mais il doit aussi se choisir des femelles en bonne santé afin de les accoupler aux mâles performants locaux ou exotiques pour améliorer leur productivité. II est certain que la maîtrise de la reproduction est indissociable dans

\section{RÉFÉRENCES}

Bauer B, Dittami J, Huber S 2009 Effects of nutritional quality during early development on body weight and reproductive maturation of guinea pigs (Cavia porcellus). Gen. Comp. Endocrin. 161, 384-389;

Chauca de Zaldivar 1999 Produccion de cuyes en crianzas familiares en Peru. In: V Congreso variant entre 1,57 pour la première mise bas, 1,92 pour la deuxième mise bas et 2,00 pour la troisième mise bas. Considérant les différentes études, nos résultats sont satisfaisants, mais il y a lieu de les améliorer par une meilleure conduite d'élevage.

Intervalle des mises bas : L'intervalle entre mises bas est de 90,2 $\pm 5,5$ jours pour les hybrides issus des croisements Bv x Bv ; il est de 89,3 $\pm 4,3$ jours pour ceux des croisements Blg x Kin. Fransolet et al (1994) ont trouvé au Gabon 74 jours; Fosto et al (1995) au Cameroun donnent 63 à 68 jours en milieu traditionnel et 86 en station. Nos résultats sont inférieurs, dus probablement au manque d'adaptation et aux conditions d'exploitation de type traditionnel.

Age à la première mise bas : L'âge moyen à la 1ère saillie est de 154,7 jours Belgique X Kinshasa et 169,25 jours Bukavu X Bukavu ; l'âge moyen à la 1ère mise bas est de 220,4 jours Belgique $X$ Kinshasa et 228,5 jours Bukavu X Bukavu. Fosto et al (1995) au Cameroun ont trouvé que l'âge moyen à la première mise bas était de $134,6 \pm 11,1$ jours (âge moyen à la première saillie : 53 jours)! Fransolet et al (1994) au Gabon ont eu la première mise bas à 77 jours après la mise au mâle ; en ajoutant 60 jours, cela donne 137 jours d'âge à la première mise bas! Nos résultats sont de loin très élevés; nous ne pouvons expliquer cela que par le manque d'adaptation et les conditions d'exploitation de type traditionnel utilisé. Le moment de la mise au mâle choisi arbitrairement est de 5 mois, conforme aux avis de Rota (1990) et de Schmidt (1980).

la pratique de celle des autres paramètres de l'élevage (alimentation, hygiène).Le cobaye, bien adapté aux conditions climatique de la République Démocratique du Congo consommant une alimentation végétale grossière non compétitive avec celle de l'homme, est un bon producteur de protéines, intéressant à élever au niveau familial ou même au niveau d'élevage intensif commercial.

Latino americano de Cuyicultura, Venezuela, Memorias..., p. 24-36 ;

Cigogna M 2000 Guide technique d'élevage $n^{\circ} 4$ : les cobayes, B.E.D.M., FUSAG, Gembloux, 8p.

Dérivaux et Ectors 1986 Reproduction chez les animaux domestiques. Paris, Acadia éditions 1141p.

Fosto J M, Ngou Ngoupayou J D et Kouonmenioc J 1995 Performances expérimentales des cobayes 
élevés pour la viande au Cameroun, Cahiers agricultures 4: 65-69;

Fransolet M C, Horlait P et Hardouin J1994 Élevage expérimental du Cobaye Cavia porcellus en région équatoriale au Gabon, Revue Elev. Méd. Vét. Pays Trop, 47 (1) : 107-111.

Hardouin J 2000 Guide technique d'élevage $n^{\circ} 3$ sur les grenouilles, Bureau pour l'échange et la distribution de l'information sur le mini-élevage (B.E.D.I.M), $8 p$;

Hardouin $J$ et Thys E 1997 Le mini-élevage, son développement villageois et l'action de BEDIM ». BIOTECHNIT, Agron. Soc-Environ. 1 (2) : $92-99$;

Havrez H 2002 Amélioration de la production de cochon d'Inde chez les petits agriculteurs de la vallée de Carhuaz (Département de Ancah, Pérou). Mémoire de fin d'études présenté en vue de l'obtention du grade d'ingénieur agronome orientation élevage, Faculté Universitaire des Sciences Agronomiques de Gembloux, inédit, 81p;

Rota A 1990 Observation concernant le rendement en carcasse des cobayes. Trav. Fin d'études. Anvers : IMT.

Salsac B 2004 Élevage des cobayes en Amérique du Sud, expérimentation: impact de 3 types d'alimentation sur la prise des poids des cobayes durant la période d'engraissement (Riobamba, Équateur), Haute École Provinciale de Hainaut Occidental, 37pages ;

Schmidt G 1980 Le cochon d'Inde. Albrecht Philler Minden, éd. Allemagne, 1980, Bibliothèque didactique, 1702. 